\title{
Smoking Cessation in Primary Care Clinics
}

Jeffrey M. Sippel, MD, MPH, Molly L. Osborne, MD, PhD, Wendy Bjornson, MPH, Bruce Goldberg, MD, A. Sonia Buist, MD

OBJECTIVES: To document smoking cessation rates achieved by applying the 1996 Agency for Health Care Policy and Research (AHCPR) smoking cessation guidelines for primary care clinics, compare these quit rates with historical results, and determine if quit rates improve with an additional motivational intervention that includes education as well as spirometry and carbon monoxide measurements.

DESIGN: Randomized clinical trial.

SETTING: Two university-affiliated community primary care clinics.

PATIENTS: Two hundred five smokers with routinely scheduled appointments.

INTERVENTION: All smokers were given advice and support according to AHCPR guidelines. Half of the subjects received additional education with spirometry and carbon monoxide measurements.

MEASUREMENTS AND MAIN RESULTS: Quit rate was evaluated at 9-month follow-up. Eleven percent of smokers were sustained quitters at follow-up. Sustained quit rate was no different for intervention and control groups ( $9 \%$ vs $14 \%$; [OR] 0.6 ; 95\% [CI] 0.2, 1.4). Nicotine replacement therapy was strongly associated with sustained cessation (OR 6.7; 95\% CI 2.3, 19.6). Subjects without insurance were the least likely to use nicotine replacement therapy $(p=.05)$. Historical data from previously published studies showed that $2 \%$ of smokers quit following physician advice, and additional support similar to AHCPR guidelines increased the quit rate to $5 \%$.

CONCLUSIONS: The sustained smoking cessation rate achieved by following AHCPR guidelines was $11 \%$ at 9 months, which compares favorably with historical results. Additional education with spirometry did not improve the quit rate. Nicotine replacement therapy was the strongest predictor of cessation, yet was used infrequently owing to cost. These findings support the use of AHCPR guidelines in primary care clinics, but do not support routine spirometry for motivating patients similar to those studied here.

KEY WORDS: clinical trial; smoking cessation; AHCPR guidelines; spirometry; nicotine replacement therapy. J GEN INTERN MED 1999;14:670-676.

Received from the Department of Medicine and the Division of Pulmonary Medicine, Oregon Health Sciences University, Portland, Ore (JMS, MLO, ASB); the Department of Family Medicine, Oregon Health Sciences University, Portland, Ore (BG); and the Tobacco-Free Coalition of Oregon, Portland, Ore (WB).

Address correspondence and reprint requests to Dr. Osborne: Associate Dean of Students, Oregon Health Sciences University, 3181 SW Sam Jackson Park Blvd., Box UHN-67, Portland, OR 97201.
$\mathrm{C}$ igarette smoking, the leading cause of preventable illness in the United States, is responsible for $\$ 50$ billion in annual health care expenditures for smokingrelated illnesses. ${ }^{1}$ Given the significant morbidity, mortality, and economic burden caused by smoking, the Agency for Health Care Policy and Research (AHCPR) published clinical guidelines for smoking cessation in 1996, making specific recommendations for primary care clinicians, smoking cessation specialists, and administrators. ${ }^{2}$ The major recommendations were to screen all patients in primary care clinics for tobacco use, advise patients who smoke tobacco to quit, and provide support and assistance throughout the cessation process. Theoretical program costs for these recommendations ranged from $\$ 1,500$ to $\$ 6,000$ per life saved, depending on the type and intensity of intervention used. ${ }^{3}$

The AHCPR guidelines, based on meta-analyses of published smoking cessation trials, ${ }^{3}$ were designed to encourage consistent, effective methods for promoting smoking cessation. Since the guidelines were released in 1996, no studies of their effectiveness when applied to primary care patient populations have been published. We therefore chose to document the usefulness of these guidelines by implementing AHCPR recommendations to identify all smokers and provide intervention in two communitybased family practice clinics at Oregon Health Sciences University (OHSU) and compare these results with historical controls. ${ }^{4}$ As smokers frequently are not motivated to quit smoking at the time of screening, ${ }^{5,6}$ we also proposed to test the hypothesis that adding a motivational intervention, consisting of routine lung function screening and education, would improve motivation to quit, quit attempts, and quit rate when compared with AHCPR recommendations alone. Evaluating the AHCPR guidelines in a primary care setting and determining whether a specific motivational intervention improved patient outcomes were our project goals.

\section{METHODS}

A program was implemented in two OHSU family practice clinics to identify all smokers among routinely scheduled outpatients. All smokers were invited to participate in the research project, and participants were randomized to control or intervention groups. Both groups received advice and cessation information according to AHCPR guidelines, ${ }^{2}$ but only the intervention group received the motivational intervention, consisting of spirometry and carbon monoxide analysis, interpretation, and education. Outcomes, including quit rates, attempt rates, and motivational stage, ${ }^{5,6}$ were compared at follow-up 
(9 months) between the control and intervention groups. The study protocol was approved by the OHSU Institutional Review Board, and written, informed consent was obtained from all subjects.

\section{Smoker Identification}

Two community-based family practice clinics with similar demographic characteristics located in the Portland metropolitan area were selected as study sites. At the time of patient registration, a colored card was stamped with each patient's identification and placed on the clinic encounter form. Cards were stamped for all regularly scheduled adult patients but not for non-Englishspeaking patients, children under the age of 18 years, or walk-in patients whose case was considered emergent by the clinic's triage nurse. At patient check-in, the nurse would ask if the patient smoked cigarettes regularly. Patients who responded yes were asked if they would volunteer to participate in a research project involving smokers. All patient responses were circled on the colored card, which was then placed in a collection box, regardless of the patient's responses. We were therefore able to track the total number of patients screened and classify them as nonsmokers, smokers willing to participate in the study, or smokers unwilling to participate in the study. If a patient made more than one clinic visit during the study period, only the results of the first screening were counted.

\section{Enrollment}

Subject Recruitment. Patients who identified themselves as regular smokers and stated their willingness to participate in the study were approached by a member of the study staff, who was on-site 30 hours weekly during the enrollment period. The study staff was either the principal investigator or a research assistant, neither of whom was an employee of the family practice clinics. Patients were informed that their participation was voluntary and not required by the clinic; the clinic would provide all patients with information about smoking cessation regardless of participation in the study; and participation in the study did not constitute automatic enrollment in a smoking cessation program. Written, informed consent was obtained.

Questionnaires. All participants completed a standardized questionnaire assessing motivational stage classification, ${ }^{6}$ nicotine dependence (Heaviness of Smoking Index), ${ }^{7}$ and medication use and demographic information. Questionnaires were administered by the study staff.

Randomization to Intervention Versus Control Groups. Questionnaires were numbered consecutively at each clinic throughout the study period. Subjects receiving odd-numbered questionnaires were selected as the inter- vention group, and those receiving even-numbered questionnaires were selected as the control group. Subjects were enrolled in chronologic order based on time of check-in. The nurses performing patient check-in were blinded to the questionnaire numbers. As four to six nurses conducted patient check-ins independently and simultaneously at each clinic, it is unlikely that any given patient would be preferentially enrolled into either study arm.

\section{Advice to All Smokers}

Smoking cessation programs were established at both clinic sites in accordance with AHCPR guidelines. ${ }^{2}$ The programs were administered by the study staff, who completed intensive training in smoking cessation methods at the Lung Health Study clinic in Portland. The participant's motivational stage was determined by asking two standard questions: (1) Do you have serious intentions to quit smoking within the next 6 months?, yes or no; and (2) Do you have serious intentions to quit smoking within the next 30 days?, yes or no. ${ }^{5}$ The "precontemplative" stage was defined as no intention to quit within 6 months; the "contemplative" stage, as intention to quit within 6 months but not within 30 days; and the "preparation" stage, as intention to quit within 30 days. All participants received a uniform message encouraging them to quit smoking and an individual cessation plan based on their motivational stage. Precontemplative and contemplative subjects received brief counseling (3 minutes) that included a National Institutes of Health self-help pamphlet, ${ }^{8}$ a listing with 13 community-based cessation programs and support groups, and the name and telephone number of the study staff member to request further assistance when desired. Patients in the preparation stage received all of the above and additional counseling (10 minutes) that included review of the self-help pamphlet, an on-site educational question-and-answer session, ${ }^{2,9,10}$ solicitation of a quit date, clinic or telephone follow-up 1 week and 4 weeks after quit date, encouragement to use nicotine replacement therapy (NRT), not supplied by the study, and study staff assistance to obtain NRT through the participant's insurance plan, if covered.

\section{Motivational Intervention}

For the intervention group, spirometry was performed and $\mathrm{CO}$ measurements were obtained. They then received a uniform educational interpretation of these results. The intervention took 10 to 15 minutes to perform. All tests were performed by the study staff, who completed spirometry training at the Lung Health Study clinic in Portland. Spirometry was performed with equipment that met American Thoracic Society requirements (Nellcor Puritan Bennett Spirometer, Model PB-100, Pleasanton, Calif). Standard reference values by Crapo were used to calculate percentage of predicted values for forced expiratory 
volume in one second $\left(\mathrm{FEV}_{1}\right)$ and forced vital capacity (FVC). ${ }^{11}$ Each subject performed repeated spirometry maneuvers until three acceptable tracings were obtained. Carbon monoxide level was measured using equipment that met FDA requirements (Discover Carbon Monoxide Monitor, MultiSpiro, Inc., San Clemente, Calif). A level above $5 \mathrm{ppm}$ was considered to reflect current smoking. The study staff gave the subjects an educational message that varied depending on spirometry results (normal or obstructed). Subjects were then encouraged to ask questions about their test results.

\section{Follow-up}

Subject contacts made as part of the smoking cessation program were performed by the study staff according to AHCPR guidelines. According to motivational stage, contacts might have included solicitation of a quit date, clinic or telephone follow-up 1 and 4 weeks after the quit date, and additional contacts if requested by individual subjects. All subjects received 6-month telephone followup to determine the primary and secondary study outcomes. A research assistant blinded to the subject's randomization arm conducted the 6-month follow-up independently of contacts made for smoking cessation efforts. The primary outcome variable was smoking cessation rate. Cross-sectional (sustained) quit rate was defined as a successful quit that occurred during the study period and was ongoing at the time of follow-up. Relapse was defined as a successful quit period of at least 24 hours duration during the study period that was followed by a return to smoking. Secondary outcome variables were quit attempts and change in motivational stage at follow-up. Follow-up was considered complete after successful telephone contact or after unsuccessful telephone contact and both of the following: a working telephone number was unavailable from computer records and alternate contact person; and at least two follow-up questionnaires were sent via mail. Follow-up is reported as the number of days elapsed from study entry to completed follow-up.

\section{Historical Controls}

Identification of smokers and providing advice and support for cessation should be a routine part of practice in primary care clinics; therefore, we felt it would be inappropriate to withhold such information from smokers identified through this research project. Because we did not have a group of patients who were identified as smokers but not offered cessation advice, direct comparisons were made only between the intervention and control groups, both of whom received smoking cessation advice according to AHCPR guidelines. Historical information is available from previously published studies of smoking cessation trials, which we have included for comparison. ${ }^{4,12-14}$

\section{Statistical Methods}

On the basis of data published from a study of similar design, we assumed sustained quit rates in the control group would be approximately 10\%, and that the motivational intervention would result in an approximate doubling of the quit rate. ${ }^{13} \mathrm{~A}$ sample size of 91 in each group would detect an increase in quit rate from $10 \%$ to $25 \%$ ( $\alpha=0.05$, power $=0.8$ ). We therefore chose 200 subjects as our enrollment goal.

Statistical analysis was performed using JMP software (SAS Institute, Cary, NC). Categorical variables (gender, race, employment status, prior quit attempts, insurance status, and motivational stage) are reported as percentage possessing the given characteristic. Continuous variables (age, cigarettes smoked per day, pack-years, length of follow-up, and lung function) are reported as the mean $\pm \mathrm{SE}$. Categorical variables were compared using the $\chi^{2}$ statistic, and continuous variables were compared using the Student's $t$ test. ${ }^{15}$ Logistic regression analysis adjusted for age and gender was performed to calculate odds ratios for likelihood of quit attempts and sustained smoking cessation at follow-up by subject characteristics. All $p$ values are two-sided, with statistically significant meaning a $p$ value $<$.05. If follow-up data were not available for a given subject, a conservative assumption was made that the subject was currently smoking and in a precontemplative stage.

\section{RESULTS}

\section{Patient Screening}

A total of 1,038 patients were screened between $\mathrm{Au}-$ gust 1 and October 15, 1996 (Fig. 1). Fifty-two percent (538) were nonsmokers, 35\% (360) were regular cigarette smokers, and 13\% (140) had unknown smoking status (the screening card was returned incomplete). Of the 360 smokers, 31\% (111) declined participation for unspecified reasons, and 69\% (249) agreed to participate in the study. Forty-four of the 249 subjects were not randomized because they left the clinic before study enrollment was completed and did not return. Therefore, 205 smokers

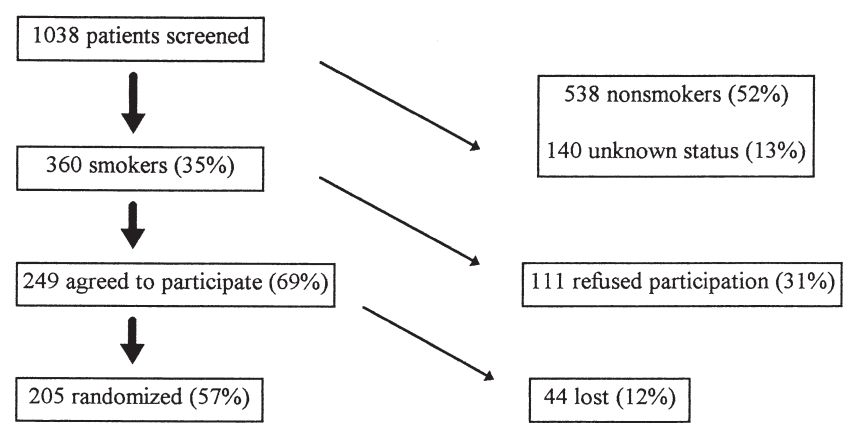

FIGURE 1. Results of clinic screenings. 
were entered into the study and randomly assigned to the control or intervention groups.

\section{Subjects}

The study population smoked an average of 20 cigarettes daily, had a 29-pack-year history, and began smoking at a mean age of 16 years. One hundred two subjects were randomized to the control group, and 103 to the intervention group. There were no significant differences between the groups in age, gender, race, employment status, level of education, or insurance status (Table 1). Several features known to predict lower rates of smoking cessation, including number of cigarettes smoked daily, prior quit attempts, and motivational stage, were also well matched between groups. The mean $\mathrm{FEV}_{1}$ in the intervention group was $87 \%$ predicted, and the mean $\mathrm{FEV}_{1}: \mathrm{FVC}$ ratio was $76 \%$. Abnormal lung function was found in a minority of subjects: $23 \%$ had an $\mathrm{FEV}_{1}: \mathrm{FVC}$ ratio less than $70 \%$. Spirometry could not be performed adequately in five subjects. The 114 smokers who declined participation were slightly older than those who participated $(42.6 \pm$ 1.2 vs $38.6 \pm 0.9, p=.01)$, whereas gender $(57.5 \%$ vs $62.4 \%$ female, $p=.39$ ) and insurance status $(9.7 \%$ vs $13 \%$ uninsured, $p=.10$ ) were no different.

\section{Participation Rates for Smoking Cessation Program}

Of 205 randomized subjects, 73 (36\%) were in the preparation stage at study entry. Sixty $(83 \%)$ of the 73 ac- cepted follow-up planning, which included solicitation of a quit date, clinic or telephone follow-up 1 and 4 weeks after the quit date, and further contacts as requested by individual subjects. There was no difference in participation rate between clinic sites. All subjects were given information about access to community-based cessation programs (some free, some telephone-based, most costing money). Three the of 205 subjects reported using this information during the study period. Only 23 (27\%) of 86 subjects who made quit attempts or quit during the study period used NRT. Of those who did not use NRT, 58\% stated cost was the reason. Subjects without insurance were the least likely to use NRT ( $p=.05)$. Use of NRT did not correlate with gender, number of cigarettes smoked daily, employment status, level of education, income, or motivational stage.

\section{Outcomes: Quit Rates, Quit Attempts, and Motivational Stage}

Mean length of follow-up was $260 \pm 45$ days, and the follow-up rate was $84.4 \%$. Twenty-eight percent of the subjects quit smoking at some time during the study period, and $11 \%$ were sustained quitters at the end of follow-up. The mean length of abstinence for sustained quitters was $139 \pm 18$ days, whereas the mean length of abstinence for those who relapsed was $18 \pm 3.5$ days. Cross-sectional (sustained) quit rate was no different between the control and intervention groups (Table 2). There was a trend for subjects in the intervention group to be more likely to make at least one quit attempt during the

Table 1. Baseline Demographic Characteristics by Study Group

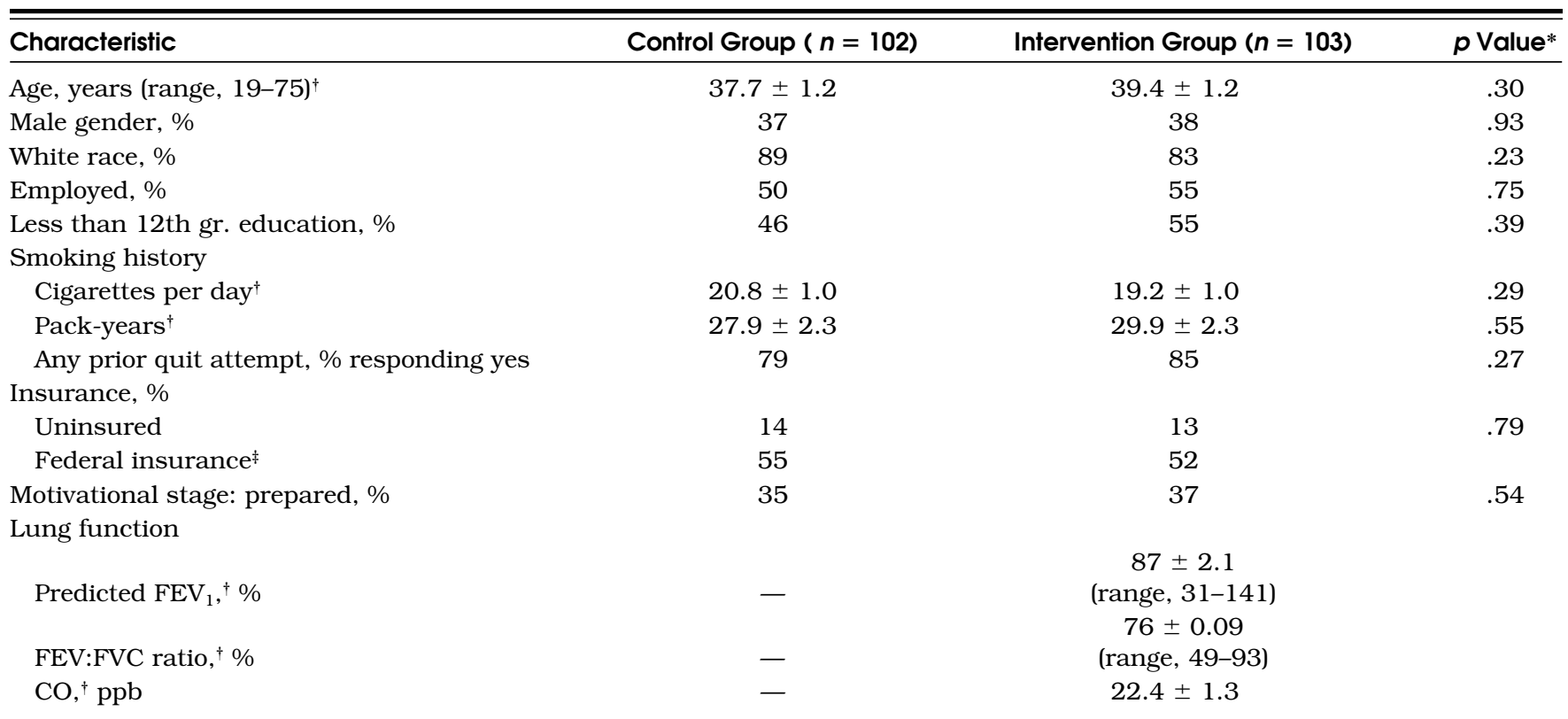

*Two-sided $\mathrm{p}$ values based on analysis of variance for age, cigarettes smoked per day, and pack-years, and Pearson's $\chi^{2}$ test statistic for all other comparisons.

${ }^{\dagger}$ Data are expressed as mean $\pm S E$.

$¥$ Federal insurance includes Medicare, Medicaid, or Oregon Health Plan. 
Table 2. Outcomes by Study Group

\begin{tabular}{|c|c|c|c|c|}
\hline Outcome & $\begin{array}{l}\text { Control Group } \\
\quad(n=102)\end{array}$ & $\begin{array}{l}\text { Intervention Group } \\
\quad(n=103)\end{array}$ & $\begin{array}{l}\text { Unadjusted } \\
\text { Odds Ratio* }\end{array}$ & Confidence Interval \\
\hline \multicolumn{5}{|l|}{ Quit rate, \% } \\
\hline Made at least 1 quit attempt during study & 36 & 48 & 1.6 & $0.9,2.8$ \\
\hline \multicolumn{5}{|l|}{ Exit motivational stage, $\%$} \\
\hline Precontemplative & 45 & 37 & & \\
\hline Lost to follow-up, \% & 18.6 & 12.6 & 0.7 & $0.3,1.4$ \\
\hline
\end{tabular}

* Odds ratio for the intervention group, based on logistic regression analysis.

${ }^{\dagger}$ Two-sided p values on Pearson's $\chi^{2}$ test statistic for the three motivational stages.

study period than those in the control group (odds ratio [OR] $1.6 ; 95 \%$ confidence interval $[\mathrm{CI}] 0.9 ; 2.8$ ), but this was of borderline statistical significance $(p=.09)$. Motivational stage at exit did not differ between groups (Table 2). Nicotine replacement therapy was strongly associated with sustained smoking cessation (OR 6.7; 95\% CI 2.3, 19.6), whereas number of cigarettes smoked daily, time to first cigarette, education, and insurance status correlated negatively with sustained quitting during the study period (Table 3). Abnormal spirometry results did not correlate with smoking cessation. There was no relation between smoking cessation and age (OR 1.6; 95\% CI 0.2, 13.2), weight (OR 0.8; 95\% CI 0.07, 6.9), or gender (OR 0.6; 95\% CI $0.2,1.4)$. Unadjusted odds ratios and confidence intervals for characteristics shown in Table 3 were nearly identical to those adjusted for age and gender and therefore are not shown. Because the study population was $86 \%$ white, we were unable to discern a relation between race and smoking cessation.

\section{DISCUSSION}

This randomized clinical trial showed that routine identification of all smokers in a primary care clinic, along with advice and support according to AHCPR guidelines, resulted in a sustained quit rate of $11 \%$ at 9 -month follow-up. Although there was a trend for subjects who received the motivational intervention to be more likely to make at least one quit attempt during the study period than the control group, sustained quit rates and motivational stage were no different at follow-up.

The motivational intervention tested in this study consisted of additional education with personal spirometry and carbon monoxide results. It did not involve either behavior modification techniques or mandatory pharmacotherapy. More quit attempts were made in the intervention group, but the sustained quit rate was no different. These results are similar to those of two other studies performed before the AHCPR guidelines were developed. Jamrozik and colleagues studied 2,110 smokers and reported that the addition of $\mathrm{CO}$ monitoring to a program that included verbal and written antismoking advice increased quit rates from $15 \%$ to $17 \%(p<.05) .{ }^{12}$ Segnan et al. studied 923 smokers and showed that adding spirometry to a program with repeated counseling sessions improved quit rates from $5.5 \%$ to $6.5 \%$, which was not statistically significant. ${ }^{14}$

However, one should not interpret these studies to mean that there is no role for spirometry in smokers. In the present study, composed of highly nicotine-addicted smokers of lower socioeconomic status, routine spirometry was not beneficial. Other studies have shown benefit in different populations. Risser and Belcher reported that the addition of spirometry and $\mathrm{CO}$ measurements to a smoking cessation program improved quit rates from $11 \%$ to $24 \%$ at 1 year. ${ }^{13}$ However, their study population was fundamentally different from this one. All subjects were participants in a voluntary health fair who had received mailed invitations encouraging their participation. These recruitment methods very likely selected more motivated smokers for whom a motivational intervention would be expected to have a greater effect. In contrast, our study attempted to identify and enroll all smokers among routinely scheduled outpatients in two family practice clinics, and used spirometry as motivation for all smokers, not only those who were more highly motivated. Although spirometry and CO monitoring may be helpful for more motivated smokers, as suggested by the Risser and Belcher study, the lack of benefit of spirometry seen here suggests that patient support, behavioral modification, and pharmacotherapy are essential components of effective smoking cessation programs for patients similar to those studied here.

There are limitations to this study that affect the generalizability of our conclusions. First, the system used to identify smokers was imperfect: $13 \%$ of the screening cards were returned incomplete, generally as a result of inadvertent omissions by the check-in nurses. Given the number of nurses involved with check-ins (an average of 4 at each site for any given half-day clinic period), the overall $87 \%$ completion rate was considered acceptable. Second, only $82 \%$ of patients who agreed to participate in the 
Table 3. Odds Ratios for Sustained Quitting During the Study Period

\begin{tabular}{|c|c|c|c|}
\hline Characteristic & Variable for Odds Ratio & Adjusted Odds Ratio* & $95 \%$ Confidence Interval* \\
\hline Spirometry & Performed & 0.6 & $0.2,1.4$ \\
\hline Abnormal spirometry & Yes & 0.6 & $0.1,2.7$ \\
\hline Clinic site & Gabriel Park & 0.7 & $0.3,1.7$ \\
\hline Successful 24-hr quit prior to study period & Yes & 4.3 & $1.5,15.7$ \\
\hline NRT used during study period ${ }^{\dagger}$ & Yes & 6.7 & $2.3,19.6$ \\
\hline$>10$ Cigarettes daily & Yes & 0.17 & $0.06,0.5$ \\
\hline First daily cigarette within $30 \mathrm{~min}$ & Yes & 0.4 & $0.16,1.01$ \\
\hline \multirow[t]{2}{*}{ Education } & Beyond high school & Reference & \\
\hline & 11 th Grade or less & 0.1 & $0.09,0.6$ \\
\hline \multirow[t]{2}{*}{ Insurance status } & Third-party & Reference & \\
\hline & Federal ${ }^{\ddagger}$ & 0.15 & $0.04,0.4$ \\
\hline Motivational stage & Preparation & 1.9 & $0.7,5.4$ \\
\hline
\end{tabular}

* Based on logistic regression analysis adjusted for age and gender.

${ }^{\dagger} N R T$ indicates nicotine replacement therapy.

${ }^{\ddagger}$ Federal insurance includes Medicare, Medicaid, or Oregon Health Plan.

study were actually enrolled. As there was only one study staff member at each clinic site, some patients left the clinic before enrollment was completed when there was a backlog of subjects. Third, the check-in nurses, but not the study staff, were blinded to the randomization procedure, and the possibility of nonrandom assignment of subjects existed. However, Table 1 demonstrates that key features known to predict rates of smoking cessation, including number of cigarettes smoked daily, prior quit attempts, motivational stage, race, employment status, level of education, and insurance status were well matched between groups, suggesting the randomization procedure worked very well. Finally, follow-up data were obtained via self-report from telephone interviews with study subjects, which could bias results toward overreporting of abstinence. The Lung Health Study followed 5,887 smokers and reported a small but significant bias toward overreporting abstinence, by about $4 \%$, when comparing selfreport to biochemical verification as the gold standard. ${ }^{16}$ If the bias toward overreporting were $4 \%$ in this study population, the sustained quit rate would have been $10.8 \%$ rather than $11.2 \%$, which does not affect the overall study results.

The clinics selected for this study were felt to be representative of community-based primary care practices in the Portland metropolitan area. However, a wide degree of variation exists among primary care populations, influenced by the sociodemographics of the surrounding community. University-affiliated clinics may also have a different patient profile than nonaffiliated clinics even within the same community. The demographic profile in Table 1 highlights important characteristics of the patients studied here. Specifically, most smokers (including nonparticipants) were female. Participants were also fairly young, poor, and with low socioeconomic standing as reflected by self-report and insurance status. Although conclusions drawn from this study provide useful information to most primary care practitioners and administrators, they may not be generalizable to all patient populations, given these unique features.

The primary care clinics used in this study did not have organized smoking cessation efforts in place prior to this study's inception. The only antismoking resource uniformly available for patients was physician advice, and neither clinic had standardized smoking cessation pamphlets or community-based cessation program information available. As baseline clinic quit rates prior to this study are unknown, we have only historical results by which to gauge the effectiveness of the AHCPR guidelines as applied to these clinics. Historical data show that physician advice alone results in a quit rate of about $2 \%$ per year, based on studies with a combined total of 14,438 smokers; and additional advice and support increase the quit rate to about $5 \%$, based on studies with a combined total of 6,466 smokers. ${ }^{4}$ There are no unique patient demographic characteristics, health care provider characteristics, or available antismoking resources at these clinics that would suggest quit rates should be substantially higher than in historical controls. The overall sustained quit rate of $11 \%$ in this study compares favorably to these historical controls.

The AHCPR guidelines strongly emphasize the use of NRT for patients making a quit attempt. ${ }^{2}$ However, implementation of such recommendations is dependent on resources, such as nicotine patches or gum, being readily available for patients. Data published elsewhere suggest that as few as $11 \%$ of health plans cover NRT. ${ }^{2}$ Although the use of NRT was strongly encouraged among all participants in this study, it was not provided free of charge. As a consequence, only one in four smokers who made quit attempts used NRT. Most subjects cited cost as the deterrent, and insurance plans did not cover the cost in most cases. In fact, subjects without insurance were the least likely to use NRT ( $p=.05)$. Sustained quit rates were three times higher among subjects who used NRT, with an OR of 6.7 favoring cessation. Had NRT been universally 
available and free of charge, it is likely that the overall quit rate would have been much greater. These findings support the AHCPR recommendations that NRT be offered to all smokers attempting to quit.

This study was designed to detect a difference in quit rate of $15 \%$ between intervention and control groups. This level of difference was chosen on the basis of results from the previously mentioned study by Risser and Belcher. ${ }^{13}$ Quit rates for the intervention and control groups were similar, and the wide confidence interval (Table 2) does not suggest a trend toward increased quit rate in the intervention group. There was, however, a trend for more smokers in the intervention group to make at least one quit attempt during the study period (OR 1.6; 95\% CI 0.9, 2.8). Quit attempts are known to correlate with smoking cessation. ${ }^{5,6}$ The advice and information received by these smokers, though not initially successful, may contribute to future smoking cessation by reinforcing messages from other sources. ${ }^{17}$

Less than $2 \%$ of smokers in this study used resources outside their clinic when attempting to quit, despite being provided with information about access to 13 community-based smoking cessation programs. This finding strongly supports AHCPR recommendations that all primary care clinics develop on-site smoking cessation resources rather than relying solely on outside programs. Community-based resources are invaluable for many smokers, but they should not be used as a substitute for clinic-based cessation efforts in primary care clinics with demographic characteristics similar to those studied here.

In summary, this randomized clinical trial showed that implementation of AHCPR smoking cessation guidelines in two primary care clinics resulted in a sustained quit rate of $11 \%$ at 9 -month follow-up. Although there was a trend for subjects who received a motivational intervention to be more likely to make at least one quit attempt during the study period than the control group, sustained quit rates at follow-up were no different. The study also revealed that NRT was the strongest predictor of successful quitting, yet was used infrequently because of its cost; and community-based smoking cessation resources were used even less frequently. Widespread implementation of clinic-based smoking cessation programs that follow AHCPR guidelines will have measurable health benefits for smoking patients.

This project was funded by the American Lung Association of Oregon and the American Academy of Family Practice. The authors thank Gary Sexton, PhD, for assistance with data analysis; Mary Joyce for her invaluable efforts as a research assis- tant; Drs. Lyle Fagnan and Sam Richard for their assistance at the clinic sites; and Joyce Riha-Linik and Michael Tennison for technical support.

\section{REFERENCES}

1. Cigarette smoking among adults-United States, 1992, and changes in the definition of current cigarette smoking. MMWR. 1994;43:342-6. [Erratum. MMWR. 1994;(43):801-3].

2. The Agency for Health Care Policy and Research Smoking Cessation Clinical Practice Guideline. JAMA. 1996;275:1270-80.

3. Cromwell J, Bartosch WJ, Fiore MC, Hasselblad V, Baker T. Costeffectiveness of the clinical practice recommendations in the AHCPR guideline for smoking cessation. Agency for Health Care Policy and Research. JAMA. 1997;278:1759-66.

4. Law M, Tang JL. An analysis of the effectiveness of interventions intended to help people stop smoking. Arch Intern Med. 1995;155: 1933-41.

5. Prochaska JO, DiClemente CC, Norcross JC. In search of how people change: applications to addictive behaviors. Am Psychol. 1992;47:1102-14.

6. DiClemente CC, Prochaska JO, Fairhurst SK, Velicer WF, Velasquez MM, Rossi JS. The process of smoking cessation: an analysis of precontemplation, contemplation, and preparation stages of change. J Consult Clin Psychol. 1991;59:295-304.

7. Heatherton TF, Kozlowski LT, Frecker RC, Fagerstrom KO. The Fagerstrom test for nicotine dependence: a revision of the Fagerstrom tolerance questionnaire. Br J Addict. 1991;86:1119-27.

8. Clearing the Air. How to Quit Smoking . . . and Quit for Keeps. Bethesda, Md: National Institutes of Health; 1993. NIH publication 94-1647.

9. Strategies to Control Tobacco Use in the United States: A Blueprint for Public Health Action in the 1990s. Smoking and Tobacco Control Monographs No. 1. Rockville, Md: Department of Health and Human Services; 1991.

10. Manley MW, Glynn TJ. How to Help Your Patients Stop Smoking. A Manual for Physicians. Bethesda, Md: National Cancer Institute; 1992. NIH publication 93-3064.

11. Becklake M, Crapo RO. Lung function testing: selection of reference values and interpretive strategies. Am Rev Respir Dis. 1991; 144:1202-18.

12. Jamrozik K, Vessey M, Fowler G, Wald N, Parker G, Van Vunakis H. Controlled trial of three different antismoking interventions in general practice. BMJ. 1984;288:1499-503.

13. Risser NL, Belcher DW. Adding spirometry, carbon monoxide, and pulmonary symptom results to smoking cessation counseling: a randomized trial. J Gen Intern Med. 1990;5:16-22.

14. Segnan N, Ponti A, Battista RN, et al. A randomized trial of smoking cessation interventions in general practice in Italy. Cancer Causes Control. 1991;2:239-46.

15. Fleiss JL. Statistical Methods for Rates and Proportions. New York, NY: John Wiley and Sons; 1981.

16. Murray RP, Connett JE, Lauger GG, Voelker HT. Error in smoking measures: effects of intervention on relations of cotinine and carbon monoxide to self-reported smoking. The Lung Health Study Research Group. Am J Public Health. 1993;83:1251-7.

17. Manley MW, Epps RP, Glynn TJ. The clinician's role in promoting smoking cessation among clinic patients. Med Clin North Am. 1992;76:477-94. 